\title{
Terrestrial Planet Finder Coronagraph Instrument Design
}

S. B. Shaklan, K. Balasubramanian, D. Ceperly, J. J. Green, D. J. Hoppe, O. P. Lay, P. D. Lisman and P. Z. Mouroulis

Jet Propulsion Laboratory, California Institute of Technology, 4800 Oak Grove Dr., Pasadena, CA 91009

email: stuart.b.shaklan@jpl.nasa.gov

Abstract. We describe the baseline TPF-C instrument design as of October 2005.

\section{Introduction}

For the past 2 years, NASA has invested substantial resources to study the design and performance of the Terrestrial Planet Finder Coronagraph (TPF-C). The work, led by the Jet Propulsion Laboratory with collaboration from Goddard Space Flight Center and several university and commercial entities, encompasses the starlight suppression system, observatory design, performance modeling, materials characterization, primary mirror studies, and a significant technology development effort including a high-contrast imaging testbed that has achieved 1e-9 contrast in a laboratory experiment (Trauger et al. (2005)). TPF-C papers covering the full range of these topics appear in the proceedings of the 2005 SPIE meeting (conferences 5905, 5867, and 5895) and are too numerous to list in this brief manuscript.

\section{Requirements}

A group of U.S.-based scientists is presently preparing the high-level science requirements. The notional requirements call for the capability to detect $\sim 30$ terrestrial planets in the habitable zone (HZ) assuming all stars have one such planet (and likewise, $\sim 3$ planets if the probability of occurrence of a terrestrial planet around any star is 10\%).

The science requirements lead to a challenging set of performance requirements. The reflected visible/NIR light from a half-illuminated earth-like planet at 1 AU from a solartype star is $\sim 10^{10}$ times fainter than the starlight. For a star at a distance of $10 \mathrm{pc}$, the planet appears 100 mas from the star. Studies of observing completeness (Brown (2004), Brown (2005)) and our own mission completeness studies find that to meet the science requirements for terrestrial planet detection, TPF-C should achieve the performance requirements listed in Table 1.

Table 1: TPF-C Coronagraph Contrast Error Budget Requirements

\begin{tabular}{lcl}
\hline Parameter & Requirement & Comment \\
\hline Static Contrast & $6 \times 10^{-11}$ & Coherent terms \\
Contrast Stability & $2 \times 10^{-11}$ & Thermal + Jitter \\
Instrument Stray Light & $1.5 \times 10^{-11}$ & Incoherent light \\
Inner Working Angle & $4 \lambda / \mathrm{D}_{\text {long }}$ & 57 mas at $\lambda=550 \mathrm{~nm}, \mathrm{D}_{\text {long }}=8 \mathrm{~m}$ \\
Outer Working Angle & $48 \lambda / \mathrm{D}_{\text {short }}$ & $1.5 \operatorname{arcsec}$ at $\lambda=550 \mathrm{~nm}, \mathrm{D}_{\text {short }}=3.5 \mathrm{~m}$ \\
\hline
\end{tabular}



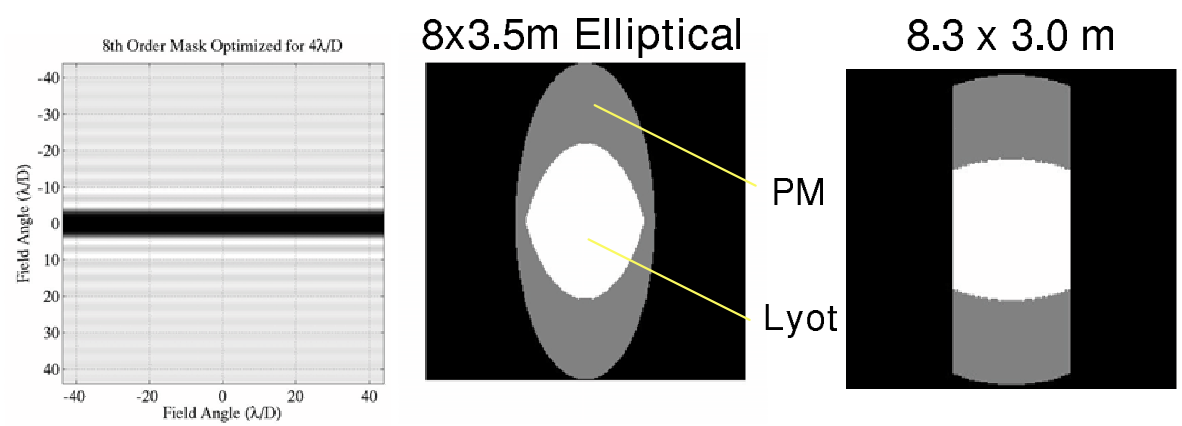

Figure 1. Left: the transmission function of the 8th-order coronagraph mask. Middle: the original $8 \times 3.5 \mathrm{~m}$ elliptical paerture and Lyot stop for operation at $4 \lambda / \mathrm{D}$. Right: the $8.3 \times 3 \mathrm{~m}$ 'bandaid' design and corresponding Lyot stop.

"Contrast" refers to the level of light scattered by the instrument, relative the incident star light. The contrast stability is the parameter that determines the limiting signal-tonoise ratio (SNR). At a level of $2 \times 10^{-11}$, a planet having contrast $10^{-10}$ ( $\left.\operatorname{mag}=25\right)$ can be detected with an instrument-limited $\mathrm{SNR}=5$. The inner working angle (IWA) is the closest point to the star where a planet can be detected. Extensive modeling (e.g. Green \& Shaklan (2003)) has shown that for a Lyot coronagraph, it is impractical to work within 3-4 /D ( the third Airy ring) because as one removes diffraction at smaller working angles, the Lyot aperture is reduced while aberration sensitivity increases. Our inner working angle of $4 \lambda / \mathrm{D}$ represents a compromise between the required resolution $(\sim 60$ mas), the largest aperture that can fit in an existing launch shroud, and the engineering requirements at the IWA. The outer working angle (OWA) is fundamentally limited by the number of actuators in the deformable mirror (DM). An OWA of 1.5 arcsec is achieved by spanning the short axis of the primary mirror with 96 actuators.

\section{Instrument Design}

\subsection{Apertures}

The aforementioned models show that monolithic apertures are far superior to segmented apertures in a high-contrast coronagraph. Segments lead to significant throughput losses at the segment edges, but more importantly relative piston motions of the segments scatters light across the coronagraph 'dark hole' and must be controlled to picometer levels. Monolithic apertures are required but are limited in their dimension by the launch vehicle shroud. Engineers at Ball Aerospace developed the concept of an elliptical aperture coronagraph (Noecker et al. (2003)). The long axis delivers the required resolution while the total area provides adequate sensitivity. We have recently evolved this approach from its original elliptical shape to a nearly rectangular aperture (it has the shape of a common bandage), as shown in Fig. 1

The advantages of the 'band aid' aperture are two-fold. First, in the presence of a linear occulter mask, the rectangular shape improves Lyot stop efficiency. For the case shown, it improves from $34 \%$ (elliptical) to $44 \%$ (rectangular). Second, the shape allows one to employ a so-called 'bar-code' shaped-pupil mask (Vanderbei et al. (2004)). These masks are straightforward to build and model, and can be made broad-band and polarizationindependent. 
Table 2. Coronagraph Trades

\begin{tabular}{|c|c|c|c|c|}
\hline Property & Occulter / Lyot & Shaped Pupil & PIAA & Visible Nuller \\
\hline Practical IWA & $3.0-3.5 \lambda / \mathrm{D}$ & $3.5-4.5 \lambda / \mathrm{D}$ & 2.0-3.0 $\lambda / \mathrm{D}$ ? & $3.0-3.5 \lambda / \mathrm{D}$ \\
\hline Stop Efficiency & $40-60 \%$ & $25 \%$ & $\sim 100 \%$ & $40-60 \%$ \\
\hline $\begin{array}{r}\text { Immediate Discovery } \\
\text { Space }\end{array}$ & $\begin{array}{c}>70 \% \\
\text { Constrained by } \\
\text { occulter }\end{array}$ & $\begin{array}{c}>70 \% \text { Constrained } \\
\text { by limited } \\
\text { diffraction shaping }\end{array}$ & $\begin{array}{c}>90 \% \text { Constrained } \\
\text { by field dependent } \\
\text { aberrations }\end{array}$ & $\begin{array}{c}<25 \% \text { Constrained } \\
\text { by fringe pattern } \\
\text { and fiber } \\
\text { attenuation } \\
\end{array}$ \\
\hline $\begin{array}{r}\text { Minimum Primary } \\
\text { Mirror Dimensions } \\
\end{array}$ & $8 \times 3.5 \mathrm{~m}$ & $8 \times 3.5 \mathrm{~m}$ & $<6 \times 3.5 \mathrm{~m}$ & $8 \times 3.5 \mathrm{~m}$ \\
\hline SM Despace Stability & $20 \mathrm{~nm}$ & $20 \mathrm{~nm}$ & $1 \mathrm{~nm} ?$ & $1 \mathrm{~nm}$ \\
\hline $\begin{array}{r}\text { Implementation } \\
\text { Complexity } \\
\end{array}$ & Low & Low & Moderate & High \\
\hline Critical Issue & $\begin{array}{c}\text { Complex errors } \\
\text { induced at a } \\
\text { focal plane may } \\
\text { limit broadband } \\
\text { utility }\end{array}$ & $\begin{array}{c}\text { Interaction } \\
\text { between high- } \\
\text { angle diffraction } \\
\text { and high-frequency } \\
\text { speckles }\end{array}$ & $\begin{array}{l}\text { Fabrication of } \\
\text { optical } \\
\text { components. } \\
\text { Diffraction? }\end{array}$ & $\begin{array}{l}\text { Implementation } \\
\text { complexity }\end{array}$ \\
\hline
\end{tabular}

\subsection{Coronagraphs}

Several coronagraph forms have been considered, including band-limited Lyot coronagraphs (Kuchner \& Traub (2002)), shaped pupils (Kasdin et al. (2003)), remapped pupils (Guyon et al. (2005)), a visible nulling instrument (Mennesson et al. (2003)), and a vortex-mask coronagraph (Palacios (2005)). Four-quadrant phase masks are not considered because their 2nd-order dependence on wave front tilt does not sufficiently suppress starlight. Vortex masks appear to have good aberration rejection and high throughput but have not been modeled to the same level of detail as band-limited masks. The baseline design includes accommodation for Lyot coronagraphs and shaped-pupils. Both can be implemented using a filter wheel approach to select different masks for different purposes (e.g. improved discovery space vs. deeper contrast over a restricted space).

Table 2 outlines the trade-offs between the various concepts. PIAA (pupil remapping) is the most promising but least mature concept. PIAA offers $100 \%$ throughput and $100 \%$ discovery space for planets outside the 2nd Airy ring. However fabrication of components and potential diffraction limitations (Vanderbei (2005)) must be explored before this approach is deemed viable. Laboratory tests in a high-dynamic range coronagraph are underway.. The baseline Lyot coronagraph is a linear 8th-order mask (Kuchner et al. (2005), Shaklan \& Green (2005)). The 8th-order null is effective at filtering low-order aberrations that will result when the primary mirror sees thermal gradients and when the secondary mirror moves relative to the primary. Compared to a 4th-order null (e.g. the visible nuller), the 8th-order mask is 1-2 orders of magnitude less sensitive to changes in aberration content at the IWA. It also offers excellent discovery space - a planet can be detected with nearly equal efficiency beyond the IWA except for a central strip running orthogonal to the mask oscillations. The mask will likely be fabricated in HEBS glass (Trauger et al. (2005)) as this continuous-tone approach is the least polarization sensitive. We are currently addressing achromatization of the mask densification process. Binary mask implementations (e.g. notch-filter masks, (Kuchner \& Spergel (2003)) have severe polarization and chromaticity issues (Lay et al. (2005)). The bar-code shaped-pupil mask shares low aberration sensitivity and large discovery space with the 8th-order mask. It has comparable throughput at the IWA but worse throughput for planets at $>\sim 6 \lambda / \mathrm{D}$. It is the simplest form of coronagraph to implement.

\subsection{Wave Front Control (WFC)}

Our WFC system employs two high-performance Xinetics DMs (Ealey \& Trauger (2004)) arranged in a Michelson interferometer configuration. This allows independent control of 
both phase and amplitude over the dark hole, but the wave front correction has wavelength dependence that does precludes full correction of reflectivity and phase-induced amplitude errors over a broad spectral band. We are also considering ways to implement sequential DMs to help conjugate far-from-pupil optics. This approach may also eliminate the need for the Michelson beamsplitter and wedges which are likely to introduce additional chromatic and polarization issues. The DM format is $96 \times 96$ actuators on a $1 \mathrm{~mm}$ pitch. Thermal stabilization of the DMs is crucial - sub-Angstrom wave front stability is required during the extent of our 'set-and-forget' observing scenario (Shaklan et al. (2005)).

\subsection{Other Aspects}

The optical design includes an afocal cylindrical telescope that reimages the short axis of the telescope across the full width of the DM (thus forming a square image of the primary aperture on the DM). This increase the outer working angle, significantly expanding the detection space of detection of Jovian planets. The design also includes two orthogonal polarization channels. This was originally implemented for three reasons. First, the slightly different aberration content of each polarization leaked around the 4th order mask resulting in unacceptable static contrast. Second, binary masks are not simultaneously effective in both polarizations. These issues are remedied through the use of 8th order masks and by employing HEBS masks rather than binary ones. Third, the secondary channel offers full redundancy in case of DM or detector failure. We are currently considering eliminating the second channel and readdressing the redundancy issues.

\section{Acknowledgements}

The work described here has been performed at the Jet Propulsion Laboratory, California Institute of Technology, under a contract with the National Aeronautics and Space Administration.

\section{References}

Brown, R.A. 2004 ApJ 607, 1003

Brown, R.A. 2005 ApJ 624, 1010

Green, J.J., \& Shaklan, S.B. 2003 Proc. SPIE 5170, 38

Guyon, O. et al. 2005 ApJ 622, 744

Ealey, M.A \& Trauger, J.T. 2004 Proc. SPIE 5166, 172

Kasdin, N.J. et al. 2003 ApJ 582, 1147

Kuchner, M.J. \& Traub, W.A. 2002 ApJ 570, 900

Kuchner, M.J. \& Spergel, D.N. 2003 ApJ 594, 617

Kuchner, M.J. Crepp J. \& Ge J. 2005 ApJ 628, 466

Lay, O.P., et al. 2005 Proc. SPIE 5905 (in press)

Mennesson, B. et al. 2003 Proc. SPIE 4860, 32

Noecker, N.C, Woodruff, R.A., \& Burrows C. 2003 Proc. SPIE 4860, 72

Palacios, D. 2005 Proc. SPIE 5905 (in press)

Shaklan, S.B. \& Green, J.J. 2005 ApJ 628, 474

Shaklan, S.B. et al. 2005 textitProc. SPIE 5905 (in press)

Trauger, J.T. et al. 2005 Proc. SPIE 5905 (in press)

Vanderbei, R.J., Kasdin, N.J, \& Spergel, D.N. 2004 ApJ 615, 555.

Vanderbei, R.J. 2005 ApJ (submitted) 